\title{
TEOLOGÍA, FILOSOFÍA Y CIENCIA ANTE LA CUESTIÓN DEL HOMBRE: UNA APROXIMACIÓN DESDE EL PLANTEAMIENTO DE MAX SCHELER
}

\author{
THEOLOGY, PHILOSOPHY AND SCIENCE \\ IN FRONT OF THE HUMAN QUESTION: AN \\ APPROACH FROM MAX SCHELER POSITION
}

EMILIO JIMÉNEZ ${ }^{1}$

Recibido: 7 de noviembre de 2016. Aprobado: 20 de febrero de 2017.

${ }^{1}$ Pontificia Universidad Católica del Ecuador, Facultad Eclesiástica de Ciencias Filosófico-Teológicas, Escuela de Filosofía, Quito-Ecuador, (emiliocjm@hotmail.com). 



\title{
TEOLOGÍA, FILOSOFÍA Y CIENCIA ANTE LA CUESTIÓN DEL HOMBRE: UNA APROXIMACIÓN DESDE EL PLANTEAMIENTO DE MAX SCHELER
}

\author{
THEOLOGY, PHILOSOPHY AND SCIENCE \\ IN FRONT OF THE HUMAN QUESTION: AN \\ APPROACH FROM MAX SCHELER POSITION
}

Emilio Jiménez

Palabras clave: filosofía, teología, antropología, unidad, Scheler

Key Words: theology, philosophy, anthropology, unity, Scheler

\section{RESUMEN}

La tensión entre la Filosofía, la Teología y la Ciencia en el caso concreto del tema y el problema del hombre, es una cuestión históricamente dada. La problemática humana, cuestión susceptible de encararse filosófica, teológica o científicamente, ha impulsado la navegación teórica de filósofos, teólogos y científicos. Max Scheler afirma que no hay una idea unitaria del problema del hombre; en su defecto hay tres grandes círculos de ideas: griega, judeocristiana y "cientí- fica". En este escenario surge la curiosidad por explorar la razón de esta falta de unidad a partir de postular el hecho que con frecuencia triunfa la exclusión entre protagonistas de estos tres grandes círculos de ideas antes que, como se pudiera esperar, la complementariedad y la armonía. Tanto la "ciencia" como la teología recurren a la "autoridad", el paradigma de la comunidad científica o la Biblia, pero las grandes preguntas formuladas alrededor del fenómeno humano reclaman 
incesantemente horizontes de respuesta más allá de un pretendido agotamiento de los mismos en la ciencia experimental o la teología, es decir, hubo y habrá lugar para la filosofía, más aún, resulta imperativo que lo haya, si por otra parte, una idea unitaria es imposible donde cualesquier dogmatismo acrítico se impone o intenta imponerse. No parece factible encarar la complejidad de lo humano sin el examen, la reflexión y la crítica a partir de los aportes de la ciencia, ni tampoco en ausencia de postulados cuya razonabilidad depende de la autoridad; en suma, el camino hacia la comprensión del hombre es una empresa de carácter filosófico en tanto se da en la línea de una perspectiva e idea unitaria.

\section{SUMMARY}

The tension among the Philosophy, the Theology and the Science, in short case the point and the human problem; it is a question historically given. The human problems, is a sensitive question to face philosophical, theological or scientifically, it have been promoted the theoretical navigation of philosophers, theologians and scientists.

Max Scheler affirmed the absence of a unitarian idea of the human problem, in their defect, he remarks, there are three big circles of ideas: Greek, Judeo-Christian and Scientific idea. In this context emerge the curiosity for to explore the reason of this lack of unit from postulate the fact that with frequently triumph the exclusion between protagonists of this three big circles of ideas, as what would be expected, the complementary and the harmony. The "Science" and the Theology Both appeal to the "authority", in one hand the science community paradigm or Holy Scripture on the other hand; but the big questions formulated around human phenomenon they claim incessantly forecast of answer beyond of a pretended exhaustion of them, in the science experience this side and the Theology on the other, that is to say, there was and there will be place to philosophy, even more, it is imperative that it exists, if otherwise, a unitary idea is impossible where whatever uncritically dogmatism is imposed or it tries to imposed. It does not seems feasible to confront the complexity of de human without the examination, the reflection and the critic from contributions of "Science", not even in absence of postulates whose reasonability depend on the authority; in short, the way toward human understanding is it an enterprise of philosophical nature, as long 
as, it happens in a perspective and unitarian idea and this supposes dialogue,

self-criticism, reflection and analysis.

\section{INTRODUCCIÓN}

El universo del hombre, sus temáticas y problemáticas asociadas, han sido un desafío no solo para la Filosofía, no solo para la Teología, no solo para la "Ciencia"; lo muestra el planteamiento de Scheler acerca de la existencia de tres grandes círculos de ideas. La pregunta por "el ser del hombre", en tanto se formula filosóficamente, esto es, ¿Qué es el hombre? permite ubicar y distinguir, de cara a la misma, el aporte de saberes distintos; pero ubicar y distinguir estos saberes es siempre un ejercicio en el horizonte del diálogo, más allá de posibles exclusiones a causa de posturas dogmáticas. Ubicar y distinguir no equivale en ningún caso a excluir pero, en la práctica, más que "ubicación" y "distinción" lo que se ha dado es una suerte de "exclusión" de saberes. ¿Cuál es la razón de esta "exclusión"? en las líneas que siguen se percibirá una aproximación a esta inquietud; desde luego, y ya que todo lo que se ha podido "decir con sentido" en relación al hombre está presente, como señala el planteamiento del autor citado, en tres grandes círculos de ideas, judeocristiana, griega y "científica", se encontrarán elementos vinculados a la cuestión acerca de las condiciones de posibilidad de una visión unitaria que la Filosofía desde su propia naturaleza podría ofrecer.

Al momento de plantear aspectos que permitan ubicar y distinguir los aportes de la Teología, la Filosofía y la "Ciencia", este artículo recurre a lo planteado por Kant en su obra El Conflicto de las Facultades donde clarifica la posición de la Filosofía respecto de la naturaleza de facultades a cuya base está el principio de la "autoridad".

La pregunta por el hombre, una constante en la historia del pensamiento, se vuelve un imperativo en la actualidad; mucho más, si con el tiempo se han consolidado distintos aportes desde saberes ubicables y distinguibles. La Antropología Filosófica, como se verá, una disciplina reciente respecto de otras disciplinas en la Filosofía como la Ontología, la Gnoseología o la Ética, tiene, tal como deja sentado Scheler, entre sus tareas la de mostrar cómo es la estructura del ser humano. Si lo que se ha dicho en relación al universo de lo humano es ubicable en ámbitos como la Teología, la Filosofía y la "Ciencia", es que, en tal razón, el hombre descubre rasgos comunes con 
la totalidad de los seres pero, al mismo tiempo, deja ver elementos propios y constitutivos sin los cuales carecería de sentido una empresa como la de Scheler, a saber, establecer el puesto del hombre en el ser.

\section{2. ¿UNA VISIÓN UNITARIA?}

"La misión de una antropología filosófica es mostrar exactamente cómo es la estructura fundamental del ser humano" (Scheler, 1938, pág. 129)

Uno de los autores reconocidos como de innegable influencia en la reflexión filosófica acerca del hombre es Max Scheler ${ }^{1}$, alemán nacido en Munich en 1875 y fallecido en 1928, conocedor de los principios de la fenomenología de Husserl y firme propulsor de una idea unitaria del hombre que pueda integrar elementos tanto de una antropología científica, como de una antropología filosófica y una antropología teológica. La pregunta esencial a la que se enfrenta la reflexión de Scheler se formula de forma eminentemente filosófica, a saber, ¿Qué es el hombre y cuál es su puesto en el ser? Los esfuerzos del autor en mención convergen en el anhelo de establecer rasgos propios y constitutivos del ser humano que permitan singularizarlo, esto es, identificarlo respecto de los demás seres en el cosmos. ${ }^{2}$ Para bosquejar una nueva imagen de la estructura esencial del hombre Scheler (1938) considera "un tesoro" el aporte del saber especializado que las distintas ciencias han labrado acerca del hombre. Pero aun si se reconoce el aporte de las ciencias particulares no deja de ser una preocupación en el autor mencionado la falta de integración desde una perspectiva unitaria -tarea de cuño filosófico- los aportes de la teología, la filosofía y la "ciencia" en relación a la cuestión del hombre. ${ }^{3}$

\footnotetext{
${ }^{1}$ Reflexiones recientes como la de Leonardo Polo en su libro "Quién es el hombre" dejan ver el influjo de las ideas de Scheler; aquí se retoma la idea de "el hombre un espíritu en el mundo", idea que Scheler presentará como un rasgo constitutivo del hombre en el cosmos al hablar de "categorías espirituales" que delatan un "ser espiritual" como en el caso de la categoría de sustancia.

${ }^{2}$ Se trata aquí del esfuerzo por investigar la especificidad del ser humano y, hablando en términos de la ciencia experimental, de investigar los hechos que caracterizan dicha especificidad.

${ }^{3}$ Manuel Carreira (1997) postula esta idea del modo siguiente: "Durante años he tenido la experiencia (....) de constatar cómo públicos de toda clase, desde investigadores nucleares hasta teólogos acuden a conferencias que intentan abrir ante ellos un panorama que amplía su visión, no para enfrentar un modo de conocer la realidad contra otro, sino para completar con diversas perspectivas lo que siempre será un
} 
Las investigaciones de las ciencias particulares como la biología y la botánica permiten acercarse a "las propiedades objetivas que pertenecen esencialmente al fenómeno de las cosas llamadas vivas" (Scheler, 1938, pág. 29) El hombre, en tanto ser vivo, como es evidente, comparte con los seres vivos, plantas y animales, propiedades como la nutrición, el crecimiento, la reproducción y la muerte; no obstante, la explicación del hombre no se agota en lo que de él se pueda decir cómo un ser vivo entre los demás. La pregunta ¿qué es el hombre? navega en las aguas de la Filosofía en razón de la necesidad de una incesante búsqueda tendiente a ubicar elementos propios y constitutivos de lo humano junto con la forma como se entienden estos elementos de cara a otros seres con propiedades distintas. Si tomásemos por ejemplo la propiedad de las plantas como seres vivos, una planta, en la aguda percepción de Scheler, posee en la existencia vegetativa el fenómeno primordial de la expresión, a saber, marchito, lozano, exuberante, pobre... pero "solo en el hombre se alza sobre las funciones de expresión y notificación la función de representación y denominación de signos"(Scheler, 1938, pág. 36) En efecto, si hay propiedades predicables en forma exclusiva del hombre, son estas las que entran en el espectro gnoseoló- gico de la pregunta acerca de qué es el hombre y las problemáticas derivadas.

Las conclusiones a las que llega Scheler ubican al hombre como un ser continente de todos los grados esenciales de la existencia y en particular de la vida: el grado ínfimo de lo psíquico, el impulso afectivo presente ya en las plantas y el instinto de los animales en cuanto descubren una conducta particular. Pero hay propiedades que no son de todos los seres vivos como la memoria asociativa, esta falta a las plantas como ya había advertido en su momento Aristóteles; la memoria asociativa es atribuible únicamente a los seres vivos cuya conducta se modifica lenta y continuamente en forma útil a la vida. Darwin (2014) expuso con nitidez científica el principio de la evolución biológica por la selección natural donde las especies mutan y tienen necesidad de hacerlo ante la exigencia de adaptarse al medio como condición para la sobrevivencia. Habrían razones para el extraordinario éxito del ser vivo hombre. Para empezar, una suerte de memoria biológica a favor de organismos que por ensayo y error han asimilado convenientemente aspectos ligados a su continuación en la vida. Si a un organismo le deja de ser útil para la vida un órgano en particular, este en las generaciones siguientes tenderá a desaparecer, así parecen haber evolucioconocimiento parcial e incompleto" (pag 5) 
nado las especies tal como lo sugiere la ciencia experimental y sus análisis. Ahora bien, el conocimiento del universo de lo humano, aun con la importancia de "conocer" su trayectoria en el marco de la evolución biológica, no se agota en los resultados que arroja la ciencia fáctica en cuyas descripciones aparecen aspectos como el hecho de que al hombre le corresponden "altas facultades intelectuales que le han permitido desarrollar el lenguaje articulado, agente principal de sus extraordinarios progresos" (Darwin, 2014, pág. 59) junto con la peculiaridad humana del sentimiento de lo bello.

En este sentido, la reflexión filosófica encara, además, esas particularidades del hombre como un ser que junto con manifestar el impulso, el instinto, la memoria asociativa, la inteligencia práctica, es capaz de "crear" un mundo en virtud del modo particular en el que potencialmente puede administrar, ordenar, privilegiar o disminuir lo que él mismo como ser concentra de la naturaleza. Scheler señala por ejemplo la actitud vital que, en el hombre, consiste en la mera persecución del placer y que, a su juicio, "representa una manifestación de vejez, no solo en la vida del individuo, sino también en la de los pueblos" (1938, pág. 53)

El hecho de que el hombre aparezca como un organismo dotado de propiedades adicionales al resto de organismos volvería insuficiente una des- cripción meramente biológica de él. No es suficiente la descripción biológica del hombre pues, si bien en él se revelan elementos comunes con el resto de organismos vivos, también descubre capacidades adicionales como, en el ejemplo anterior, la facultad de aislar el placer ligado a otros aspectos de la vida para dar lugar a un comer por el placer de comer o un beber por el placer de beber, relegando, deliberadamente, a un segundo plano los objetivos al servicio de la vida propios del comer y propios del beber. Es tema de análisis, reflexión y examen riguroso el hecho de que un ser como el hombre esté en capacidad de desdeñar hasta cierto punto provechos inmediatos para alcanzar de este modo otras ventajas mayores; lo que el animal no tiene, discurre Scheler, es la facultad de preferir entre los valores mismos (por ejemplo, lo útil y lo agradable) prescindiendo de los bienes concretos y singulares. Manuel Carreira (1997) amplía esta idea:

"No puede definirse el ser humano por el número de gramos de cada elemento presente en nuestro organismo, ni por la actividad físico química de las células. Ni es susceptible de comprobación experimental el valor artístico de un poema, ni el valor ético de una acción" (pag. 9)

Por lo que aparece hasta aquí, es 
posible afirmar con los planteamientos de Scheler y las ampliaciones pertinentes que las propiedades de la vida son atributos que el ser humano, como ser vivo, comparte con el resto de seres vivos. El ser humano en cuanto ser vivo tiene propiedades en común con todos los organismos vivos; lo que se pueda decir con el rigor de la ciencia experimental de un organismo vivo se podrá decir, ipso facto, del ser humano en tanto organismo vivo. La autoridad de la ciencia biológica en temas de la explicación del hombre como un organismo vivo entre los demás es, aquí, innegable. Pero, tratándose de establecer una diferencia "esencial", que la hay, esta no se da a nivel de la vida en general sino en un principio que se opone a toda vida en general asociado al fundamento supremo de las cosas y que los griegos Ilamaron razón. Scheler denomina a este principio, "espíritu"4 El hombre es el único capaz de "actos espirituales", esto es, puede realizar acciones conscientes; ve y sabe que ve, oye y sabe que oye a diferencia del animal que, por el contrario, ve sin saberlo y oye sin saberlo. El animal no se posee a sí mismo ni tampoco puede hacer suyo el medio como si puede el hombre poseerse a sí mismo y hacer suyo el medio y convertirlo en mundo. Ejemplificado esto mismo con Nietzsche, una fórmula de afirmación radical como la tragedia, sería rastreable en un ser capaz de asimilar o negar lo dado en su existencia, en categorías propiamente nietzscheanas, la voluntad de poder como un querer (aspirar) al dominio (poder) a través del arte, la representación. La condición trágica de Sísifo enseña, acerca de una de las propiedades del hombre, que a este le es posible superar el absurdo de un esfuerzo inútil apropiándose del destino, esto es, no queriendo nada distinto, no queriéndose distinto, afirmándose y en esta afirmación poder entrar activamente como un protagonista de la existencia y no tan solo como "algo" que la padece sin más. (Nietszche, 2011)

Ahora bien, si la misión de una Antropología Filosófica es mostrar cual es la estructura fundamental del ser humano, en el cumplimiento de este cometido y en atención a lo que caracteriza a la Filosofía, tendrá que tentar la idea del "todo" como algo más la suma de las partes. La totalidad de las partes del paisaje en un rompecabezas contenido en un recipiente solo es el paisaje cuando se ha podido articular. La Filosofía, la Teología y la "Ciencia" entregarían, cada una y como saberes, partes para la construcción de una comprensión unitaria de la realidad

\footnotetext{
4 "Espíritu" en Scheler tal como el mismo lo señala comprende el concepto de la razón pero también una determinada especie de intuición, la intuición de los fenómenos primarios y además una determinada clase de actos emocionales y volitivos por ejemplo la bondad, el amor, el arrepentimiento, la veneración, etc.
} 
humana que, en la opinión de Scheller, no existe. El horizonte articulador, base de la naturaleza de la actividad filosófica, es la pregunta metafísica por el qué cuyo espectro cobija no solo "hechos dados a la experiencia" sino también aqueIlo no circunscrito a ella como la cuestión de la libertad, Dios y la inmortalidad. Debemos a Kant una reflexión destacada y sistemática en torno a los límites de la razón pura teórica y constatamos por esas mismas reflexiones que la razón pura teórica no podría extralimitarse sin caer ella en contradicciones insalvables y que, al mismo tiempo, la razón pura práctica, teniendo que encarar aspectos ligados a la moral, establece "la existencia de Dios como postulado de la razón pura práctica" (Kant I, sf pag. 258) Frente a tres grandes círculos de ideas, Teología, Filosofía, "Ciencia", la Antropología Filosófica es tal en razón de la búsqueda de esa estructura que permita encarar la realidad humana desde la idea del todo que la constituye; de este modo, su actividad crítica ocurre bajo la perspectiva de la unidad llamada a forjase en la diferencia. Mostrar cómo sea la estructura del ser humano no es tarea que pueda agotarse en la "Ciencia", la Filosofía o la Teología tomadas aisladamente. Tampoco es una empresa que logre su cometido sin los aportes de la ciencia, la filosofía y la teología. Lo que hasta ahora se ha dicho y podemos decir del ser humano se ha di- cho y se dice gracias a las contribuciones de la "Ciencia", la Filosofía y la Teología. Desde la "Ciencia" y en tanto una especie entre las demás, el hombre ha evolucionado como todas las demás especies; desde la filosofía y en tanto ser particular con características propias, el hombre presenta rasgos esenciales de trascendencia, libertad y conciencia; desde la teología y en tanto sujeto de revelación, el hombre se presenta como creado a imagen y semejanza de Dios.

El ser humano y su complejidad, por lo dicho, ha permitido la navegación teórica a filósofos, teólogos y científicos: pesa, piensa, espera; el hombre pesa como cualquier piedra pero estas en comparación con aquel, en una diferencia esencial, ni piensan, ni esperan. Por eso la realidad humana toda no es explicable en términos exclusivamente físicos. De este modo, no solo ha tenido lugar la pregunta por el qué de lo humano sino igualmente la pregunta acerca del porqué y junto a ella la cuestión del cómo, sin dejar de lado la cuestión del futuro abordada en filósofos como Kant por la pregunta ¿Qué me cabe esperar? Hay aspectos en el hombre dados a la precisión de la ciencia experimental: la masa muscular, la temperatura corporal, sus parámetros biométricos, su origen. Sabemos el origen biológico y sus posibilidades en tanto ser vivo. Pero, como advierte Scheler, hay en el hombre actos espirituales 
que delatan categorías espirituales en las que este aparece distinto y por eso mismo "ubicable" dentro de la totalidad del ser. En efecto, el hombre <es>, mejor dicho, comparte con la totalidad de las cosas <el ser> pero muestra capacidades distintas y así resulta forzoso el que se lo ubique en sus particularidades. Un ejemplo puede ser la categoría de "sustancia" supuesta la cual se explica bien porque un mono huye de un plátano si lo recibe a medio pelar pero, en cambio, lo come si lo recibe enteramente pelado y aún lo pela para después comérselo si lo ha recibido sin pelar; para el animal la cosa no se ha "modificado" sino que se ha transformado en otra. Existen, pues, actos exclusivos del espíritu que, dadas sus características, plantean desafíos no solo a la ciencia, ni solo a la filosofía, ni solo a la teología.

En las consideraciones de Scheler, el hombre, es un asceta de la vida, es decir, el ser vivo que puede adoptar una conducta ascética frente a la vida. El animal al parecer es incapaz de decir un "no" deliberado a la realidad; el hombre, por el contrario, es el único ser en el universo capaz de negarse deliberadamente a un vaso de agua en el desierto aun cuando la muerte sea el precio. El hombre, evidentemente, delibera en campos donde es posible la deliberación pues y a modo de ejemplo "nadie delibera (...) sobre los solsticios y salida de los astros" (Aristóteles, 1993, pág. 67)

Scheler observa, además, que el problema "alma-cuerpo", objeto de tensiones a lo largo de varios siglos, ha perdido su rango metafísico; hoy filósofos, médicos y naturalistas que se ocupan de esta cuestión, convergen cada vez más en la unidad de una concepción fundamental. Además destaca la presencia de múltiples concepciones del hombre en la historia como la desarrollada por el sensualismo inglés cuya expresión más perfecta sería David Hume en su tratado de la naturaleza humana; igualmente la concepción del hombre como un ser dominado primordialmente por los impulsos de poderío nacida históricamente de Maquiavelo, Tomás Hobbes y los grandes políticos del estado absolutista.

Las múltiples concepciones de lo humano, nacidas de la Filosofía, la Teología y la Ciencia Particular, apelan a nociones propias con sentido y validez rigurosa dentro de su campo. La Teología, por citar un caso, acude a la noción de "revelación", que en el campo teológico no reviste mayor dificultad, sí cuando se adopta para desarrollar una filosofía evidenciando una vinculación difícil, (Fernández Hart, 2013) tal como lo constató en su momento la obra de Kant, ( 2004) el conflicto de las facultades. 


\section{EL AVANCE DE LA CIENCIA Y EL PROBLEMA DEL HOMBRE: DOS ENFOQUES}

El Décimo Octavo Congreso Mundial de Filosofía celebrado en Brighton en agosto de 1988 abordó el problema del hombre en la Filosofía. Esta cita mundial aportó, por la temática abordada, importantes luces acerca del estado de la investigación de la Antropología Filosófica. En general entregó elementos para entender mejor el fenómeno contemporáneo de la investigación del problema del hombre que involucra de forma activa a numerosas ciencias y que da como resultado un volumen amplio de conocimientos "científicos" acerca del hombre. Estos conocimientos son "particulares $^{\prime \prime 5}$ y reclaman componer un cuadro completo de la vida y la actividad del ser humano. Hay, en la percepción general de la reflexión a propósito de este congreso, dos enfoques opuestos entre sí cuando se aborda el problema del hombre, a saber, el enfoque cientificista y el enfoque anticientificista. Existe aquí un interés que lleva a los investigadores a ubicar las consecuencias de absolutizar cualesquiera de los dos enfoques y la pregunta surge entonces: ¿ofrecen absolutizados, cualesquiera de los dos, una imagen completa del ser humano? Los esfuerzos a nivel de la investigación en la Antropología Filosófica, en atención a esta última pregunta y tomando en cuenta la misma preocupación de Scheler en el sentido de la falta de una idea unitaria en relación al problema del hombre, van orientados a encarar el desafío de tentar la idea de totalidad en relación al fenómeno humano, elemento que da sentido a la adjetivación de la antropología como filosófica pues el interés por el todo como idea (no solo como la suma de las partes) es característica genuina de la Filosofía.

Los aportes del Congreso en 1988 retomaron elementos del marxismo y su tesis: "no existe más que la naturaleza y el hombre que con su actividad la transforma; la primera y decisiva peculiaridad del hombre es el trabajo transformador de la naturaleza" (Academia de Ciencias de la URSS, 1988, pág. 19) Sin embargo, más allá de que lo anterior pueda tomarse como una constatación del peso de un filósofo en la concepción contemporánea del hombre, nuevamente aparecen elementos que, vistos así, se predican como exclusivos del hombre y por eso mismo esenciales y constitutivos en la definición de lo humano. Estas cuestiones, entonces, son parte de la investigación

${ }^{5}$ La Biología, por ejemplo, estudia la estructura y la actividad vital del organismo humano en tanto organismo. 
en el campo de la antropología filosófica pues, como se ve, se busca conocer los elementos que caracterizan al hombre y únicamente al hombre. Scheler parece ser refrendado en la afirmación que "no hay razones para admitir que los animales saben y comprenden lo que hacen" (Academia de Ciencias de la URSS, 1988, pág. 68) El hombre es el animal que crea instrumentos de trabajo según un plan y propósitos definidos. La trampa de los cazadores humanos se construye como instrumento de caza existiendo la posibilidad de perfeccionarla cada vez a través de los conocimientos y las asociaciones. Pero las aves que migran de Europa a América, eligiendo la vía más cómoda y corta a través del océano no poseen conocimientos de navegantes. El hombre, a diferencia del animal, no puede hacer cosa alguna sin saber cómo hacerla, sin tener idea de su estructura. "La convicción de que los animales no poseen consciencia, no piensan ni conocen, a la que se han atenido no solo los idealistas sino también la mayoría de los realistas, es, más que un testimonio de la "arrogancia" humana, una determinada explicación si bien no muy adecuada, del hecho de que los hombres descubren, ubican y conocen de continuo cada vez más objetos nuevos...los animales repiten de generación en generación su modo de vida anterior, sin dar muestras de una capacidad medianamente notable para perfeccionarlo.

En el ser humano aparece en forma evidente la capacidad para aprender y perfeccionar aptitudes naturales a través de la educación. ${ }^{6}$ Un ejemplo elaborado por Teodor Oizeman ayuda a entenderlo: "La posición vertical al andar, indudablemente, es biológicamente inherente al hombre, al igual que el habla articulada. Pero si no se le enseña a la criatura lo uno y lo otro, jamás dominará sus aptitudes naturales" (Academia de Ciencias de la URSS, 1988, pág. 73)

En el trabajo de ubicar lo específicamente humano, tarea propia de la Antropología Filosófica, se van decantando características específicas del ser humano, entre otras, la conciencia, la libertad y la trascendencia, categorías que encierran otras manifestaciones características de lo humano asociadas a su estatuto ontológico: La muerte como suceso ineluctable, científicamente explicable pero, a la vez, como una inexorabilidad asumida, pensada y hasta deliberadamente representada.

Ante el hombre sucede la destrucción por un tsunami naturalmente dispuesto o la destrucción por una bom-

\footnotetext{
${ }^{6}$ La existencia del hombre es única, lo corporal y lo espiritual, lo consciente y lo inconsciente, lo biológicamente heredado y lo socialmente determinado, las normas culturales y la libre creatividad, las intenciones y las orientaciones axiológicas se funden en ella en un todo único.
} 
ba humanamente intencionada; el mundo de lo humano permite agrupar sus manifestaciones en las categorías de la "conciencia" (lo que sucede y el saber de lo que sucede) de la "libertad" (lo que sucede y el modo de estar ante lo que sucede) y de la "trascendencia" (lo que sucede y la forma como se formaliza en aras de comunicarlo y dotarlo de sentido).

La comprensión de la estructura fundamental del ser humano, se refleja en lo cultural, lo político, lo económico. De ahí que una de las ideas presentes en este Congreso Mundial de Filosofía haya sido la responsabilidad del hombre en el mantenimiento de la vida en el planeta.
El hombre es un ser con la capacidad de elegir y en sus elecciones trabajar en función de la vida o en detrimento de esta. El perfeccionamiento y el progreso son características específicas del hombre y de la humanidad. Un ejemplo en este sentido, tan real como preocupante, sería el que ofrece Arendt en su ensayo sobre la violencia, a propósito del perfeccionamiento de las armas con las cuales cuentan los ejércitos de hoy en estados con capacidad de vencer a un enemigo al paradójico precio de destruirse a sí mismos; dada la sofisticación de las armas nucleares la victoria de uno sería el final de los dos. (Arendt, 2013)

\section{A MODO DE CONCLUSIÓN}

Las antropologías a las que alude Scheler, teológica, filosófica y "científica", son ellas evidenciadas en la presencia de tres grandes círculos de ideas ante la pregunta qué es el hombre; lo encara la teología desde la revelación y el marco antropológico de la fe; lo analiza, critica y reflexiona la filosofía desde la razón; lo indaga y presenta la ciencia desde las posibilidades y alcance de su método particular. Cada instancia, teológica, filosófica o científica, declara cada una en sus aportes aspectos del ser humano. La complejidad del universo de lo humano ha permitido la navegación teórica del teólogo, el filósofo y el científico. Pero es cierto que, como advierte Carlos Bravo (2004), en alguna etapa de la historia, en la edad media, la teología se convirtió en la "reina de las ciencias" con la asimilación de la metafísica de Aristóteles por parte de Santo Tomás y la aceptación de sus presupuestos como evidentes en relación al conocimiento de Dios, el Mundo y el Hombre; también es cierto que con el posicionamiento de la ciencia en su versión moderna a partir de Galileo Galilei, y como se ventiló en el octavo congreso mundial de Filosofía, ha predominado en la ciencia un enfoque excluyente, "cienti- 
ficista"; pero, como instancia de permanente búsqueda, incesante reflexión y constante crítica, la Filosofía se ha planteado -y no puede dejar de hacerlo- la cuestión de una idea unitaria en relación a la temática del hombre. Esta es la tarea de una antropología filosófica, sostiene Scheler, pero, además, el problema del hombre en su complejidad amerita y vuelve imperativo una tarea de ese cuño: pensar. Lo que el tiempo ha consolidado en los ámbitos de la Teología, la Filosofía y la "Ciencia" como productos nacidos de la preocupación por la cuestión del hombre, ubicables y distinguibles, señala por lo menos que: hay rastros de verdad en el cometido del teólogo, el filósofo y el científico por lo que Teología, Filosofía y Ciencia entregan, cada una, insumos para el siempre necesario diálogo en un tema y problema de permanente análisis, reflexión y crítica: el ser humano.

Solamente establecido lo dicho, tienen lugar de pertinencia preocupaciones distintas -y por distintas complementarias- en el afán de buscar una idea unitaria del hombre, ser de especificidades y al mismo tiempo un ser de características compartidas. 


\section{REFERENCIAS BIBLIOGRAFICAS}

Academia de Ciencias de la URSS. (1988). Fernández Hart, R. (2013). Creo, luego El problema del hombre en la filosofía. Moscú: SN.

existo. Lima: Universidad Antonio Ruiz de Montoya.

Arendt, H. (1993). La Condición Humana. Buenos Aires: Paidos.

Kant, I. (2004). El Conflicto de las Facultades. Buenos Aires: Lozada.

Arendt, H. (2013). Sobre la violencia. Madrid: Alianza Eidtorial.

Kant, I. (sf). Crítica de la Razón Práctica. Madrid: Gredos.

Aristóteles, A. (1993). Etica Nicomáquea. Nietszche, F. ( 2011). Ecce Homo. Madrid: Barcelona: Gredos. Alianza .

Bravo, C. (2004). El marco antropológico de la fe. Bogotá: Universidad Javeriana.

Carreira, M. (1997). El hombre en el cosmos. Madrid: Sal Terrae.

Polo, L. (1993). Quién es el hombre. Alcalá: Rialp.

Scheler, M. (1938). El Puesto del Hombre en el Cosmos. Buenos Aires: Losada S.A.

Darwin, C. (2014). El origen del Hombre. Bogotá: Panamericana. 